\title{
MIMO Antenna Using Resonance of Ground Planes for 4G Mobile Application
}

\author{
Xing Zhao $\cdot$ Kyeol Kwon $\cdot$ Jeahoon Choi
}

\begin{abstract}
A MIMO antenna using the resonance of ground planes is proposed for $4 \mathrm{G}$ mobile application. A resonant mode is generated when the double ground planes (upper and lower) in the mobile terminal are excited as the radiator. By combining the resonant modes contributed from both the antenna element and the ground planes, the proposed MIMO antenna realizes a wideband property over LTE band 13. In addition, an inductive coil is employed to reduce the antenna volume. These approaches not only simplify antenna design but also effectively improve bandwidth and efficiency. The proposed MIMO antenna has an excellent ECC value of below 0.1 because of the nearly orthogonal radiation patterns of the two radiators. Moreover, an additional antenna is adopted to cover WiMAX, WLAN, and Bluetooth services simultaneously in frequency range from $2 \mathrm{GHz}$ to $2.7 \mathrm{GHz}$.
\end{abstract}

Key words: MIMO, 4G, ECC, Ground Planes, Inductive Coil.

\section{I . Introduction}

Fourth Generation (4G) mobile communications have received substantial attention, dominating the recent mobile communication market. The Multi-input Multi-output (MIMO) antenna is expected to be a key element in supporting $4 \mathrm{G}$ systems [1] [3]. Because of the limitation of volume in a mobile terminal, MIMO antennas usually have low efficiency and narrow bandwidth. As the biggest piece of conductor around the antenna in a mobile terminal, the ground plane has aroused much interest. It has been widely accepted that the utilization of a ground plane could substantially improve the performance of small antennas [4]. In the MIMO system, the value of Envelope Correlation Coefficient (ECC) is a key parameter in evaluating the performance of an antenna. In general, a small ECC value guarantees a good isolation performance in a MIMO system. However, isolation is not directly related to the ECC in a MIMO system with small antenna elements because of the low efficiency. So some popular approaches to enhancing isolation performance do not effectively improve ECC simultaneously [5]. The only valid method for improving ECC in a small MIMO system is to increase the pattern diversity of different antenna elements. In a MIMO system with small antenna elements and quasi-omnidirectional radiation patterns, the most effective way to lower the ECC is to increase the separation angle between the different patterns.

In this paper, a MIMO antenna designed for $4 \mathrm{G}$ mobile application is proposed. By utilizing the resonance of ground planes and an inductive coil, the proposed antenna improves both bandwidth and efficiency properties. By modifying the radiation patterns orthogonally, the MIMO antenna obtains a low ECC of below 0.1 . Moreover, an additional radiator is designed to cover WiMAX, WLAN, and Bluetooth bands with frequencies ranging from $2 \mathrm{GHz}$ to $2.7 \mathrm{GHz}$.

\section{Antenna Design and Performance}

The geometry of the proposed MIMO antenna is shown in Fig. 1.

The proposed MIMO antenna consists of three radiating elements: upper ground, lower ground, and FR4-substrate $\left(\varepsilon_{r}=4.4\right)$ with a dimension of $60 \mathrm{~mm} \times 105 \mathrm{~mm} \times$ $0.8 \mathrm{~mm}$. Radiator \#1 is located on the top edge of the substrate. Radiator \#2 is placed on the left side of the substrate. Both elements are connected to the same upper ground with a total size of $55 \mathrm{~mm} \times 95 \mathrm{~mm}$, which is printed on the top surface of the substrate. The additional Radiator \#3 is connected to the lower ground,

Manuscript received February 5, 2013 ; Revised February 27, 2013 ; Accepted March 11, 2013. (ID No. 20130205-006J)

Dept. of Electronics and Computer Engineering, Hanyang University, Seoul, Korea.

Corresponding Author : Jaehoon Choi (e-mail : choijh@hanyang.ac.kr)

This is an Open-Access article distributed under the terms of the Creative Commons Attribution Non-Commercial License (http://creativecommons.org/licenses/ by-nc/3.0) which permits unrestricted non-commercial use, distribution, and reproduction in any medium, provided the original work is properly cited. 


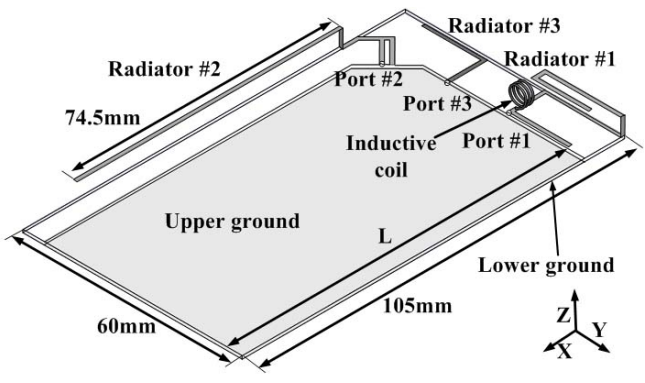

(a) Perspective view

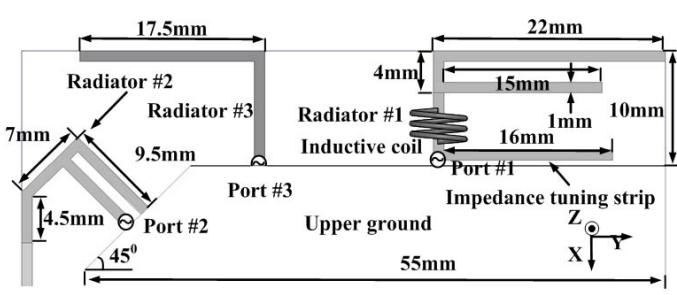

(b) Top view

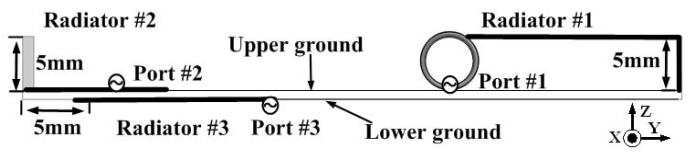

(c) Front view

Fig. 1. Geometry of the proposed MIMO antenna.

which is placed on the bottom surface. Radiator \#1 occupies a total volume of $22 \mathrm{~mm} \times 10 \mathrm{~mm} \times 5 \mathrm{~mm}$ with a strip width of $1 \mathrm{~mm}$. An additional inductive coil is made of $0.5 \mathrm{~mm}$ diameter copper wire and has three turns with a $1 \mathrm{~mm}$ pitch gap, a diameter of $5 \mathrm{~mm}$, and a height of $3 \mathrm{~mm}$. It is connected to the monopole-type radiating strip in a cascade. With three turns, the coil has an inductance of about $35 \mathrm{nH}$ [6]. Moreover, an impedance-tuning strip is located just beside the feeding point and operates as a shunt capacitance to improve impedance matching. Radiator \#2 has a long straight strip with a length of $74.5 \mathrm{~mm}$ and a width of $1 \mathrm{~mm}$. Radiator \#3 has an L-shaped strip with a total length of $27 \mathrm{~mm}$ and a width of $1 \mathrm{~mm}$.

The return losses of Radiators \#1 and \#2 with respect to variations in the length of the ground plane (L) are shown in Fig. 2(a) and (b). The resonance frequency of the mode induced by the ground planes is determined by the length (L) of the ground planes, which is approximately $0.5 \lambda$ ( $\lambda$ is the wavelength in FR-4 substrate). However, the frequencies of the modes induced by Radiators $\# 1$ and $\# 2$ are not affected by the variations in the length of the ground planes.

The corresponding current distributions on the ground planes at the resonance frequency of the ground planes $(0.752 \mathrm{GHz})$ are shown in Fig. 3. The currents on the ground planes excited by both Ports \#1 and \#2 flow mainly in the direction of the $\mathrm{x}$-axis. The maximal st-

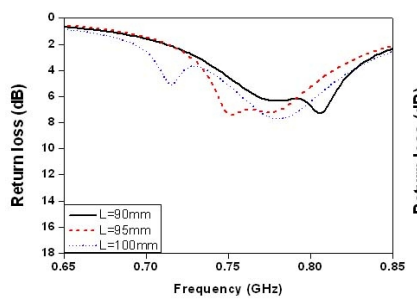

(a) Radiator \#1

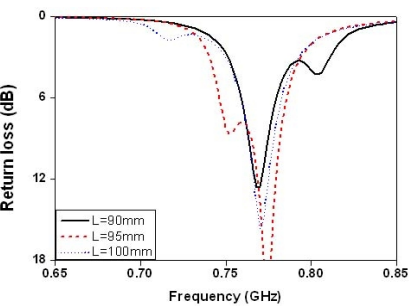

(b) Radiator \#2
Fig. 2. The return losses of Radiators \#1 and \#2 with respect to a variation in the length of the ground plane $(L)$.

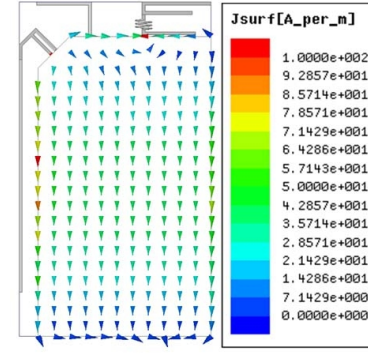

(a) Port \#1

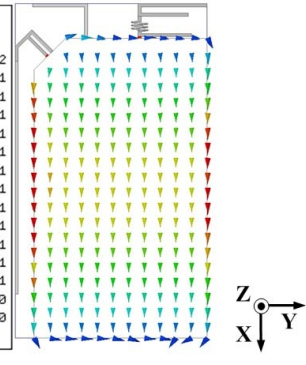

(b) Port \#2
Fig. 3. The current distributions on the ground planes at the resonance frequency of the ground planes $(0.752$ $\mathrm{GHz})$.

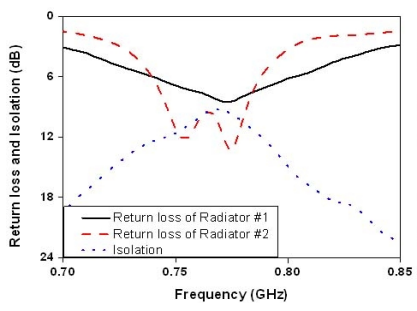

(a) Radiators \#1 and \#2 at lower LTE band 13

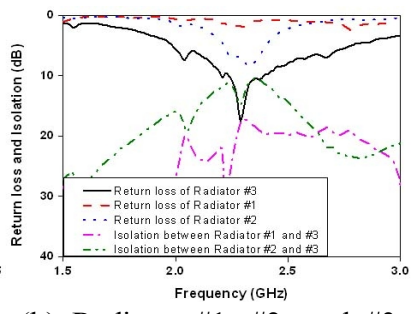

(b) Radiator \#1, \#2, and \#3 at higher band
Fig. 4. Measured return loss and isolation characteristics of the MIMO antenna.

rengths of both currents occur at the center of the ground planes. As the observation point moves from the center toward the edge of the ground planes, the strengths of the currents decrease. The quasi- $\mathrm{TM}^{\mathrm{z}}{ }_{100}$ modes are generated within the dielectric substrate between the grounds.

The measured return loss and isolation characteristics of the designed antenna are shown in Fig. 4 (a) and (b). The chart shown in Fig. 4 (a) confirms that Radiators \#1 and \#2 achieve sufficient $6 \mathrm{~dB}$ bandwidths to cover the LTE band $13(0.745 \sim 0.787 \mathrm{GHz})$. The proposed two radiators have isolation above $9 \mathrm{~dB}$ over the LTE band 13. Fig. 4 (b) confirms that Radiator $\# 3$ achieves a $6 \mathrm{~dB}$ bandwidth from $2.0 \mathrm{GHz}$ to $2.7 \mathrm{GHz}$, covering WiMAX, WLAN, and Bluetooth bands, simultaneously. Radiator \#1 does not operate at a higher band. The $6 \mathrm{~dB}$ return 


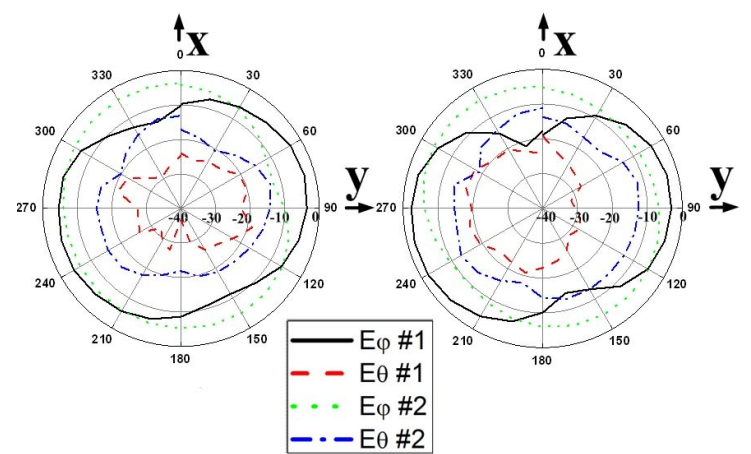

(a) $0.746 \mathrm{GHz}$

(b) $0.787 \mathrm{GHz}$

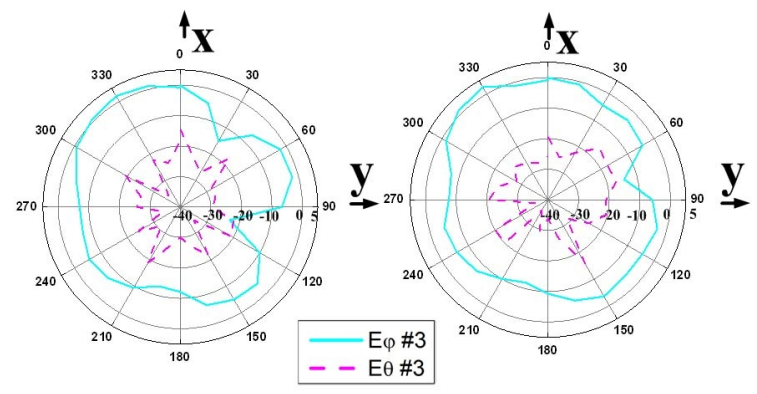

(c) $2.1 \mathrm{GHz}$

Fig. 5. Measured radiation patterns.

loss bandwidth of Radiator \#2 is narrower than that of Radiator \#3 and would not be used at a higher band. Fig. 3 shows that the isolation between Radiator \#2 and \#3 is above $10 \mathrm{~dB}$.

The measured radiation patterns are shown in Fig. 5. According to the measured results, the peak gain of Radiator \#1 is above $-1.22 \mathrm{dBi}$, and the peak gain of Radiator \#2 is above $-2.89 \mathrm{dBi}$. The peak gain of Radiator \#3 is above $1.68 \mathrm{dBi}$. As expected, the total radiation patterns of Radiators \#1 and \#2 have separation angles of around $90^{\circ}$, which lowers the ECC value.

The ECC is calculated using the measured radiation patterns, as shown in Fig. 6 [7]. The proposed MIMO antenna has an excellent ECC of below 0.1 over the LTE band 13 because of the nearly orthogonal separation angles.

The radiation efficiencies of the proposed MIMO antenna are shown in Fig. 7. As this figure shows, the
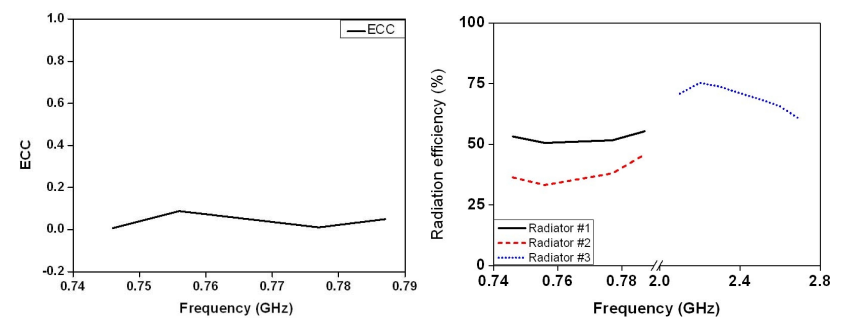

Fig. 6. ECC of proposed MI- Fig. 7. Radiation efficiencies MO antenna. of proposed MIMO antenna.

radiation efficiency of Radiator \#1 is above $50 \%$, and the radiation efficiency of Radiator $\# 2$ is above $33 \%$. The results confirm that the inductive coil in Radiator \#1 effectively improved the radiation efficiency. The radiation efficiency of Radiator \#2 is lower than that of Radiator \#1 because of the stronger opposite-directional image current on the ground planes. Moreover, the radiation efficiency of Radiator \#3 is above $60 \%$.

\section{Conclusion}

This paper proposed a MIMO antenna for $4 \mathrm{G}$ mobile applications. By utilizing the resonance of ground planes and an inductive coil, the proposed antenna achieves a simplified design in addition to improved bandwidth and efficiency. Moreover, the proposed MIMO antenna has an excellent ECC value of below 0.1 because of the nearly orthogonal radiation patterns of the two radiating elements. These properties make the proposed MIMO antenna a promising choice for $4 \mathrm{G}$ mobile applications.

This work was supported by a National Research Foundation of Korea (NRF) grant funded by the Korea government (MEST) (no. 2012-0005655).

\section{References}

[1] D. Astély, E. Dahlman, A. Furuskär, Y. Jading, M. Lindström, and S. Parkvall, "LTE: the evolution of mobile broadband," IEEE Communications Magazine, vol. 47, no. 4, pp. 44-51, 2009.

[2] B. Y. Cho, J. Y. Kim, "Novel MIMO communication scheme for enhanced indoor performance in distributed antenna systems," JKIEES, vol. 10, no. 4, pp. 263-269, 2010.

[3] S. H. Lee, J. U. Kim, "Sum-rate capacity with fairness in correlated MIMO broadcast channels," JKIEES, vol. 9, no. 3, pp. 124-129, 2009.

[4] S. R, Best, "A discussion on small antenna operating with small finite ground planes," Proc. International Workshop on Antenna Technology: Small Antennas and Novel Metamaterial, pp. 152-155, Mar. 2006.

[5] P. Hallbjörner, "The significance of radiation efficiencies when using S-parameters to calculate the received signal correlation from two antennas," IEEE Antennas Wireless Propag. Lett., vol. 4, pp. 97-99, 2005.

[6] R. Lundin, "A handbook formula for the inductance of single layer circular coil," Proc. IEEE, vol. 73, no. 9, pp. 1428-1429, Sep. 1985.

[7] J. F. Li, Q. X. Chu, and T. G. Huang, "A compact wideband MIMO antenna with two novel bent slits," IEEE Transactions on Antennas and Propagation, vol. 60, no. 2, pp. 482-489, 2012. 